\title{
24 Qualitätssicherung im optes-Projekt
}

\author{
Christian Schmidt, Gabi Reinmann
}

\subsection{Qualität und Qualitätsmanagement im Wandel der Zeit}

Das Verständnis von Qualität und Qualitätssicherung ist nicht eindeutig definiert, sondern befindet sich in einem stetigen Entwicklungsprozess und wird von unterschiedlichen Akteuren (Unternehmen, Kunden, Wissenschaft) sowie projektbezogen auf verschiedenste Weise interpretiert.

Das lateinische Wort „qualitas“, woraus sich Qualität ableitet, beschreibt zunächst einmal lediglich neutral Eigenschaften oder die Beschaffenheit eines Gegenstandes. Nach der aktuell gültigen ISO-Norm für Qualitätsmanagement wird Qualität hingegen als Maß dafür bezeichnet, inwiefern eine Ware oder Dienstleistung an sie gestellte Anforderungen erfüllen kann (DIN 2015). Diese Anforderungen können sich an einem produkt- oder fertigungsbezogenen Ansatz orientieren oder durch einen anwender- oder kundenbezogenen Ansatz festgelegt werden (Seeger und Wassermann 1998).

Das Verständnis von Qualität sowie Funktionen und Verfahren des Qualitätsmanagements sind dynamisch. So wurde etwa unter dem Begriff der Qualitätssicherung (ebd.) lange ein „Prinzip der Fehlererkennung“ (Fischer, Seeger und Wassermann 1998, S. 12) verfolgt, bei dem es um die Produktqualität ging. Später kam die Qualitätssicherung von Herstellungsprozessen durch Normen hinzu, bis sich letztendlich ein umfassender Ansatz des Qualitätsmanagements entwickeln konnte, der nicht nur die Sicherung, sondern auch die (Weiter-)Entwicklung von Qualität beinhaltet (Reinmann 2012). Auch die Institutionalisierung von Qualitätsmanagement unterliegt einem Wandel: Die Wichtigkeit von Qualitätsmanagement in Unternehmen ist unumstritten: Man erhofft sich betriebswirtschaftliche Vorteile im Wettbewerb und letztlich unternehmerischen Erfolg (Kamiske und Brauer 2002). Inzwischen sind aber auch in anderen gesellschaftlichen Bereichen Qualität und Qualitätsmanagement fester Bestandteil - so auch in Bildungsinstitutionen.

Im Zusammenhang mit Bildung (Dieninghoff 2014) bestehen allerdings Anforderungen, die weit über einen produktbezogenen Ansatz hinausgehen und entsprechende Anpassungen des Qualitätsmanagements erfordern (ebd.). In Bildungsinstitutionen kann man nicht nur Lehr-Lernmittel hinsichtlich ihrer Qualität beurteilen (dies käme einem produktbezogenen Ansatz gleich), sondern - im Prinzip - auch Lehr-Lernprozesse und -ergebnisse. Hier greifen aber gänzlich andere Ziele und Kriterien als bei Produkten und Waren. Insbesondere spielt in Bildungskontexten die Perspektive, aus der Qualität beurteilt werden soll, eine enorme Rolle. Es macht zum Beispiel an Hochschulen einen Unterschied, ob es um eine

Die Originalversion dieses Kapitels wurde revidiert. Ein Erratum ist verfügbar unter https://doi.org/10.1007/978-3-658-31279-4_32 
Qualitätsbewertung aus Sicht der Lernenden, der Lehrenden oder anderer Akteur*innen, wie etwa Studiengangsleitungen, geht.

Im optes-Verbund wurde während der Projektlaufzeit sukzessive und stets nah an den Bedürfnissen entlang des Projektverlaufs ein Konzept zur Qualitätssicherung etabliert, das in den folgenden Kapiteln näher vorgestellt wird.

\subsection{Grundsätze und Dimensionen der Qualitätssicherung in optes}

Das übergeordnete Ziel des optes-Projekts ist es, die Fähigkeiten der Studierenden zum Selbststudium während der Studieneingangsphase - dazu zählen sowohl studienvorbereitende Angebote wie Propädeutika als auch das erste Studienjahr - insbesondere in den mathematik-affinen Grundlagenfächern zu verbessern und somit die Chancen für einen erfolgreichen Studienverlauf zu erhöhen. Hierzu wurden neben elektronischen Lerninhalten zur Vermittlung mathematischer Fähigkeiten und Fertigkeiten (siehe Teil III) auch Maßnahmen zur Lernbegleitung (siehe Kapitel 18) sowie zur Kontrolle des individuellen Kenntnisstands durch formative und summative E-Assessments (siehe Kapitel 9) erarbeitet und miteinander verzahnt. Um sicherzustellen, dass dieses Zusammenspiel bestmöglich gelingt, war es essenziell, einen begleitentenden Prozess der Qualitätssicherung und -entwicklung zu realisieren.

Aufgrund der Rahmenbedingungen, die sich aus den Besonderheiten eines Projektverbunds ergeben, wurden für den Qualitätssicherungsprozess drei Grundsätze festgelegt:

1. Partizipation und Transparenz

Die Strategie zur Qualitätssicherung wurde teilprojektübergreifend gemeinsam mit allen optes-Mitarbeiter*innen erarbeitet und beschlossen.

2. Nützlichkeit

Die qualitätssichernde Maßnahmen wurden nicht um ihrer selbst willen implementiert; vielmehr wurden nur solche Maßnahmen umgesetzt, die versprachen, einen konkreten Beitrag zur Verbesserung der Qualität des optes-Angebots zu leisten.

3. Kommunikation der Ergebnisse

Sämtliche Ergebnisse aus den Maßnahmen zur Qualitätssicherung (z. B. Evaluationsergebnisse) sowie daraus gezogene Erkenntnisse wurden mit den betroffenen Verbundpartnern gemeinsam besprochen und für alle Verbundpartner ins Projekt zurückgespiegelt, sodass alle von den „Lessons Learned“ profitieren konnten. 
Die im Rahmen von optes entwickelten Maßnahmen und Angebote wurden didaktisch begründet miteinander verknüpft und über die Lernplattform ILIAS (siehe Kapitel 2) zu Lehr-Lernszenarien mit konkreten Zielen kombiniert. Entsprechend war es nicht ausreichend, die Qualitätssicherung allein auf die Dimension der Inhalte zu beziehen, sondern es mussten darüber hinaus auch die Technik sowie die Didaktik angemessen berücksichtigt werden, wobei bei der didaktischen Dimension nochmals zwischen fachdidaktischen - im Fall von optes mathematikdidaktischen - sowie allgemein-didaktischen Aspekten unterschieden wurde.

Diese drei Grundsätze und Dimensionen bildeten entsprechend die Rahmenbedingungen für sämtliche Maßnahmen zur Qualitätssicherung im Rahmen von optes.

\subsection{Gegenstandsbereich der Qualitätssicherung}

Analog zur Evaluation (siehe Kapitel 25) bestand auch bei der Qualitätssicherung die Herausforderung, unterschiedliche Arten von Ergebnissen zu berücksichtigen.

Im Rahmen von optes geht es um verschiedenste Produkte, die sich teilweise stark voneinander unterscheiden. So gibt es einerseits konkrete Lernmaterialien, aber auch Handreichungen oder Softwarelösungen. Entsprechend mussten Qualitätskriterien gefunden werden, die dieser Vielfalt an Produkten gerecht werden.

Neben den konkreten Produkten sollten aber auch die Prozesse bei der Qualitätssicherung mit berücksichtigt werden, denn sie haben indirekt Einfluss auf die Ergebnisqualität. Hier sind zum einen Prozesse im Rahmen der Projektarbeit zu nennen. Je besser die einzelnen Teilprojekte ihre Zusammenarbeit gestalten, Absprachen treffen und die Verzahnung der verschiedenen Maßnahmen bereits bei der Konzeption abstimmen, desto besser dürfte am Ende auch das Gesamtprojektergebnis sein. Zum anderen galt es auch den Lernprozess der Studierenden im Fokus der Qualitätsbestrebungen zu behalten. Hier spielt das vielfältige Support-Angebot, wie die verschiedenen Möglichkeiten des formativen E-Assessments zur Selbstdiagnostik, die Lernbegleitung oder die Arbeit mit den lernprozessbegleitenden Portfolios, eine besondere Rolle. Die Qualitätssicherung musste auch deren Komplexität und ihren Auswirkungen auf die individuellen Lernprozesse durch die Auswahl geeigneter Qualitätskriterien gerecht werden.

\subsection{Qualitätskriterien für E-Learning-Projekte}

Wie beim Qualitätsbegriff allgemein, gibt es auch speziell in Bezug auf Qualitätsmanagement für E-Learning-Angebote zahlreiche Herangehensweisen, ohne dass es einen breiten Konsens über Rahmenkonzepte, Kriterien oder Prozesse gäbe. In der einschlägigen Literatur zur Qualität beim Lernen mit digitalen Medien lässt 
sich feststellen, dass sich Qualitätsauffassungen und -kriterien weniger am Produktverständnis von Qualität, sondern eher am Lernenden (gegebenenfalls auch am Lehrenden) orientieren (z. B. Ehlers und Pawlowski 2006).

Die verschiedenen Auffassungen von Qualität, die in diesem Zusammenhang auftreten, bedingen auch unterschiedliche Formen der Qualitätsbewertung. Ehlers legt beispielsweise den Fokus auf die Perspektive des Lernenden sowie den Gegenstand und unterscheidet in der Qualitätssicherung zwischen Voraussetzungen, Lernprozessen und Lernergebnissen (Ehlers 2002), wohingegen Reinmann (2012) mit Blick auf verschiedene Ebenen und Kriterien Konzept-, Produkt- und Implementationsqualität aufführt. Dondi, Moretti und Nascimbeni (2006) wiederum unterscheiden bei den Qualitätskriterien zwischen „Learning Sources, Learning Context, and Core Learning Processes" (S. 38).

Trotz aller Unterschiede bei der Konzeptualisierung von Qualität in Bezug auf E-Learning lassen sich doch einige Qualitätskriterien identifizieren, die wiederholt auftreten und konsensfähig zu sein scheinen. Diese kamen auch im optes-Projekt zur Anwendung und wurden den oben genannten Dimensionen Inhalt, Technik und Didaktik zugeordnet.

In Bezug auf die Inhalte musste zunächst sichergestellt werden, dass diese grundlegenden formalen Kriterien entsprechen: Hierzu zählen beispielsweise die Angabe von Meta-Daten oder einheitliche Bezeichnungen. Darüber hinaus fallen fachliche Qualitätskriterien wie Richtigkeit, Relevanz oder Vollständigkeit unter diese Dimension.

Die Dimension Technik fasst Qualitätskriterien zusammen, die sich auf die technische Plattform oder die technische Umsetzung einzelner Werkzeuge beziehen. Neben Kriterien der Usability sind dies vor allem Kriterien der technischen Funktionalität wie Fehlerrobustheit oder die technische Passung zu den gewünschten Lernzwecken.

Die Dimension Didaktik bezieht sich vor allem auf die im Rahmen des optesProjekts entwickelten didaktischen Konzepte. Hier wurden formale Qualitätskriterien benannt, die teilprojektübergreifend relevant sind und sich nicht aufgabenoder zeitbezogen verändern. Ein Beispiel hierfür ist die Zweckmäßigkeit der Maßnahmen zum Erreichen der Lernziele oder eine kohärente Gestaltung. Darüber hinaus wurden für einzelne, teilprojektspezifische Angebote Kriterien aufgestellt, die spezifische Prozesse (z. B. Lernbegleitung, Selbstdiagnostik) bei der Nutzung von optes betreffen.

Auf Basis dieser Kategorisierung wurden jeweils Handreichungen für die Konzeption von Maßnahmen sowie Checklisten zur Beurteilung der Angebote erarbeitet. Als Orientierung dienten dabei im Hinblick auf die fachdidaktischen Aspekte vor allem die Einteilung zur Qualität von Mathematikunterricht von Leuders (2005) sowie in Bezug auf die technisch-gestalterische Umsetzung die Merkmale zum multimedialen Design von Niegemann et al. (2008). 


\subsection{Qualitätssicherung durch zyklisches Re-Design}

Der Kern der Qualitätssicherung im optes-Projekt folgte dem Grundgedanken einer formativen Evaluation (siehe Kapitel 25) in Kombination mit einem zyklischen Re-Design. Daraus ergibt sich ein iterativer Prozess, bei dem gemachte Erfahrungen und gesammelte Erkenntnisse genutzt werden, um projektinterne Prozesse und Projektergebnisse sukzessive zu verbessern, wobei die jeweiligen Schritte je nach dem betrachteten Gegenstand variieren.

Die wissenschaftliche Begleitung führte planmäßig und bei konkretem Bedarf Evaluationen der projektinternen Prozesse durch. Die dadurch gewonnenen Erkenntnisse wurden mit den betroffenen Partnern sowie im Gesamtprojekt besprochen; in der Folge wurden Maßnahmen erarbeitet, um die jeweiligen Bereiche der Projektarbeit zu verbessern. Der Zyklus bestand in diesem Fall aus drei Schritten (siehe Abbildung 1).

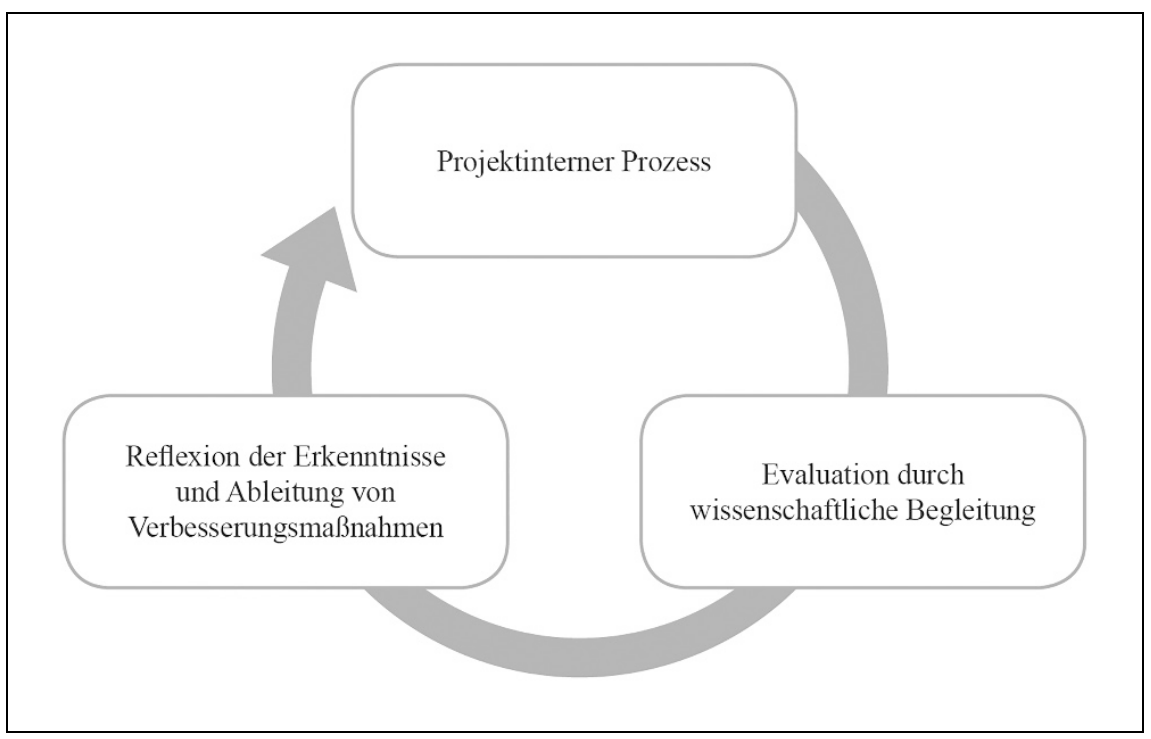

Abbildung 1: Zyklischer Prozess aus formativer Evaluation und Re-Design bei projektinternen Prozessen

In Bezug auf die entstehenden Projektergebnisse kamen weitere Schritte hinzu. Die von den jeweiligen Teilprojekten konzipierten und erarbeiteten Maßnahmen wurden zunächst bei Bedarf von projektinternen Expert*innen begutachtet und basierend auf deren Feedback überarbeitet. Die Expertenbegutachtung erfolgte fallspezifisch durch Fachleute mit technischer, mathematischer, fach- oder allgemein- 
didaktischer Expertise. Anschließend wurden die Maßnahmen an den Hochschulen im Projektverbund pilotiert und im Rahmen einer Evaluation durch die verschiedenen Zielgruppen bewertet. Die Evaluationsergebnisse, die daraus gewonnen Erkenntnisse sowie die identifizierten Optimierungsbedarfe wurden reflektiert und genutzt, um die jeweiligen Angebote weiter zu optimieren, bevor der nächste Zyklus begann (siehe Abbildung 2).

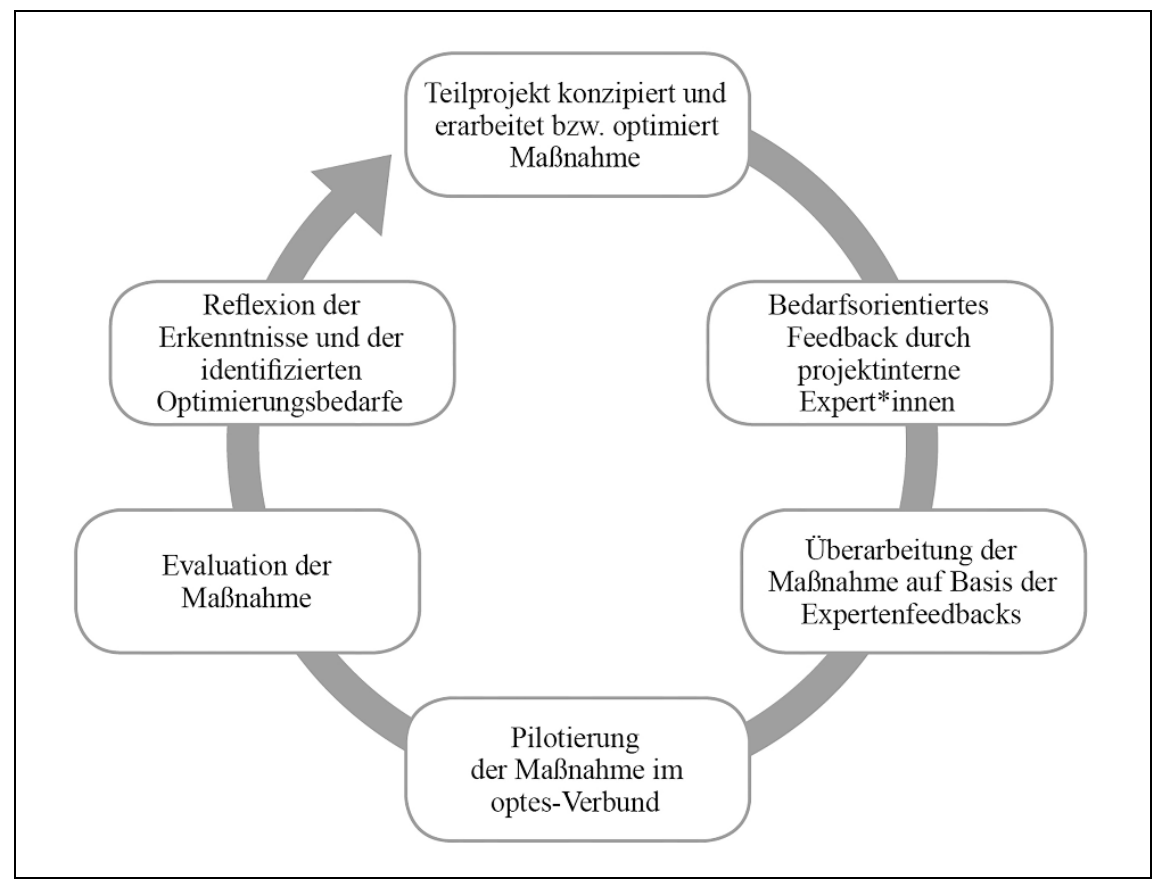

Abbildung 2: Zyklischer Prozess aus formativer Evaluation und Re-Design bei Projektergebnissen

Während die Zyklen bei projektinternen Prozessen hinsichtlich ihrer Dauer je nach konkret betrachtetem Aspekt variierten, waren die Zyklen bei den Projektergebnissen durch die möglichen Pilotzeiträume jeweils vor Studienbeginn determiniert und betrugen mit wenigen Ausnahmen ein Jahr.

\subsection{Weitere qualitätssichernde Maßnahmen}

Neben den eben skizzierten formativen Evaluationen wurden im optes-Projekt weitere Maßnahmen umgesetzt, die sich unmittelbar auf die Qualität der Projekt- 
ergebnisse auswirkten. In temporär begrenzten Arbeitsgruppen (AGs) kamen teilprojektübergreifend Projetmitarbeiter*innen mit unterschiedlichen fachlichen Hintergründen zusammen, um spezifische Themen zu bearbeiten, die zur Qualität der Projektergebnisse beitrugen. Wie dies genau aussah, soll anhand von drei Beispielen verdeutlich werden.

Ein zentraler Baustein im optes-Angebot sind die Lernzielorientierten Kurse (LoK). Im Rahmen der „AG LoK-Feedbackprozess“ haben die Mitglieder interdisziplinär und teilprojektübergreifend Qualitätsstandards für diese Kurse und die Implementation eines Feedbackprozesses erarbeitet. Entstanden ist unter anderem ein Feedbackbogen, der verschiedene Aspekte berücksichtigt: gestaltungstechnische Aspekte (z. B. Rechtschreibung, Formatierung etc.), fachdidaktische Aspekte (z. B. Einführung von Begriffen, Formulierung von Aufgabenstellungen etc.) und allgemeindidaktische Aspekte (z. B. Individualisierung, kognitive Aktivierung etc.). Der Feedbackbogen wurde erprobt und sukzessive auf sämtliche LoKs angewendet; die identifizierten Verbesserungsbedarfe wurden den Entwickler*innen zurückgespielt.

Die AG Motivation beschäftigte sich mit der Frage, wie die einzelnen optesMaßnahmen möglichst motivationsfördernd gestaltet werden können, sodass Lernende das Angebot intensiver nutzen; gleichzeitig galt es auch, Grenzen bei der Gestaltung zu erkennen. Hierzu wurden die aktuelle Nutzung des optes-Angebots umfänglich analysiert und im Verbund diskutiert sowie die bestehenden Erkenntnisse aus der Literatur aufgearbeitet. Auf Grundlage der so erzielten Ergebnisse konnten weitere Optimierungsbedarfe identifiziert und bearbeitet werden.

Im Fokus der AG Usability stand die Überlegung, wie die Nutzerfreundlichkeit der technischen Infrastruktur und auf diesem Wege die Qualität der optes-Angebote verbessert werden kann. Große Teile der optes-Maßnahmen finden innerhalb einer elektronischen Lernumgebung auf Basis der Lernplattform ILIAS statt. Entsprechend hat die Usability von ILIAS unmittelbare Auswirkungen auf die Nutzung der optes-Angebote und beeinflusst letztlich auch die von den Lernenden wahrgenommene Qualität.

Die Ergebnisse der Arbeitsgruppen unterstützten die einzelnen Teilprojekte bereits in der Phase der Konzeption und Umsetzung ihrer Angebote. Eine weitere Maßnahme, die ebenfalls im weitesten Sinne qualitätssichernd ist und an dieser Stelle der Vollständigkeit halber erwähnt werden soll, ist die summative Evaluation. Im Gegensatz zur formativen Evaluation findet diese nicht projektintern statt, sondern wird von externen Evaluator*innen abschließend durchgeführt. Auch wenn die summative Evaluation am Projektende durchgeführt wird, lässt sie sich als qualitätssichernde Maßnahme bezeichnen, denn die Ergebnisse helfen den Anwender*innen der optes-Angebote auch nach der offiziellen Projektlaufzeit, die Maßnahmen zu verbessern und weiterzuentwickeln. 


\subsection{Zusammenfassung und Fazit}

Die Qualität der optes-Angebote und ein effektiver Prozess zur Qualitätssicherung hatten im optes-Projekt einen hohen Stellenwert. Die Herausforderung bestand vor allem darin, der Komplexität des Projekts sowie den unterschiedlichen Arten von (Teil-)Projektergebnissen durch die Auswahl geeigneter Qualitätskriterien und verfahren Rechnung zu tragen. Die Kategorisierung in die Dimensionen Inhalt, Technik und Didaktik half dabei, den Überblick zu behalten.

Die Qualitätssicherung im optes-Projekt erfolgte vor allem durch einen zyklischen Prozess der formativen Evaluation und des Re-Designs. Die gewonnenen Erkenntnisse und identifizierten Optimierungsbedarfe wurden allen im Projekt zugänglich gemacht und trugen auf diese Weise zu einer sukzessiven Verbesserung der Projektergebnisse bei.

Flankierend zu diesem übergeordneten Qualitätssicherungsprozess haben sich mehrere Arbeitsgruppen mit qualitätsrelevanten Themen beschäftigt. Die Ergebnisse dieser Bemühungen unterstützten die Entwickler*innen von optes-Angeboten im Prozess der Konzeption und Pilotierung.

Es war das erklärte Ziel im optes-Projekt, eine möglichst umfassende und verzahnte Qualitätssicherung zu implementieren und diese durch regelmäßige Überprüfung und Optimierung an die Komplexität des Projekts anzupassen, um so eine möglichst hohe Qualität der Projektergebnisse zu erreichen. Inwiefern dies gelang, wird zum einen durch eine externe summative Evaluation zu bewerten sein und kann zum anderen von allen Nutzer*innen des optes-Angebots auch selbst beurteilt werden.

\section{Literatur}

Dieninghoff, M. (2014). Zur Berücksichtigung motivationaler Faktoren im Qualitätsmanagement. Wiesbaden: Springer.

DIN [Deutsches Institut für Normung e.V.] (2015). DIN EN ISO 9000:2015-11, Qualitätsmanagementsysteme - Grundlagen und Begriffe. Berlin: Beuth-Verlag.

Dondi, C., Moretti, M. \& Nascimbeni, F. (2006). Quality of e-learning: Negotiating a strategy, implementing a policy. In U.-D. Ehlers \& J. M. Pawlowski (Hrsg.), Handbook on Quality and Standardisation in E-Learning. Berlin /Heidelberg: Springer.

Ehlers, U.-D. (2002). Qualität beim E-Learning: Der Lernende als Grundkategorie bei der Qualitätssicherung. MedienPädagogik: Zeitschrift für Theorie und Praxis der Medienbildung, 5, 1-20. Verfügbar unter http://www.medienpaed.com/article/view/26/26 [10. 06.2020].

Ehlers, U.-D. \& Pawlowski, J. M. (2006). Handbook on Quality and Standardisation in ELearning. Berlin/Heidelberg: Springer.

Fischer, H., Seeger, H. \& Wassermann, O. (1998). Einführung von QualitätsmanagementSystemen. Poing: Franzis Verlag.

Kamiske, G. \& Brauer, J.-P. (2002). ABC des Qualitätsmanagements (2. Aufl.). München/Wien: Carl Hanser Verlag. 
Leuders, T. (2005). Qualität im Mathematikunterricht der Sekundarstufe I und II. Berlin: Cornelsen Scriptor.

Niegemann, H. M., Domagk, S, Hessel, S., Hein, A., Hupfer, M. \& Zobel, A. (2008). Kompendium multimediales Lernen. Berlin/Heidelberg: Springer.

Reinmann, G. (2012). Studientext Evaluation. Verfügbar unter http://gabi-reinmann.de/ wp-content/uploads/2013/05/Studientext_2012_Evaluation.pdf [10.06.2020].

Open Access Dieses Kapitel wird unter der Creative Commons Namensnennung 4.0 International Lizenz (http://creativecommons.org/licenses/by/4.0/deed. de) veröffentlicht, welche die Nutzung, Vervielfältigung, Bearbeitung, Verbreitung und Wiedergabe in jeglichem Medium und Format erlaubt, sofern Sie den/ die ursprünglichen Autor(en) und die Quelle ordnungsgemäß nennen, einen Link zur Creative Commons Lizenz beifügen und angeben, ob Änderungen vorgenommen wurden.

Die in diesem Kapitel enthaltenen Bilder und sonstiges Drittmaterial unterliegen ebenfalls der genannten Creative Commons Lizenz, sofern sich aus der Abbildungslegende nichts anderes ergibt. Sofern das betreffende Material nicht unter der genannten Creative Commons Lizenz steht und die betreffende Handlung nicht nach gesetzlichen Vorschriften erlaubt ist, ist für die oben aufgeführten Weiterverwendungen des Materials die Einwilligung des jeweiligen Rechteinhabers einzuholen.

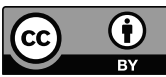

